\title{
Geometrical Hall effect and momentum-space Berry curvature from spin-reversed band pairs
}

\author{
Max Hirschberger $\odot,{ }_{1,2, *}$ Yusuke Nomura $\odot,{ }^{2}$ Hiroyuki Mitamura $\odot,{ }^{3}$ Atsushi Miyake, ${ }^{3}$ Takashi Koretsune $\odot,{ }^{4}$ Yoshio Kaneko, \\ Leonie Spitz, ${ }^{2, \dagger}$ Yasujiro Taguchi $\odot,{ }^{2}$ Akira Matsuo, ${ }^{3}$ Koichi Kindo, ${ }^{3}$ Ryotaro Arita $\odot,{ }^{1}$ \\ Masashi Tokunaga, ${ }^{3}$ and Yoshinori Tokura ${ }^{1,2,5}$ \\ ${ }^{1}$ Department of Applied Physics and Quantum-Phase Electronics Center, The University of Tokyo, Bunkyo-ku, Tokyo 113-8656, Japan \\ ${ }^{2}$ RIKEN Center for Emergent Matter Science, Wako, Saitama 351-0198, Japan \\ ${ }^{3}$ Institute for Solid State Physics, The University of Tokyo, 5-1-5 Kashiwanoha, Kashiwa, Chiba 277-8581, Japan \\ ${ }^{4}$ Department of Physics, Tohoku University, Aoba-ku, Sendai, Miyagi 980-8578, Japan \\ ${ }^{5}$ Tokyo College, The University of Tokyo, Bunkyo-ku, Tokyo 113-8656, Japan
}

(Received 7 November 2020; accepted 5 January 2021; published 25 January 2021)

\begin{abstract}
When nanometric, noncoplanar spin textures with scalar spin chirality (SSC) are coupled to itinerant electrons, they endow the quasiparticle wave functions with a gauge field, termed Berry curvature, in a way that bears analogy to relativistic spin-orbit coupling (SOC). The resulting deflection of moving charge carriers is termed the geometrical (or topological) Hall effect. Previous experimental studies modeled this signal as a real-space motion of wave packets under the influence of a quantum-mechanical phase. In contrast, we here compare the modification of Bloch waves themselves and of their energy dispersion due to SOC and SSC. Using the canted pyrochlore ferromagnet $\mathrm{Nd}_{2} \mathrm{Mo}_{2} \mathrm{O}_{7}$ as a model compound, our transport experiments and first-principles calculations show that SOC impartially mixes electronic bands with equal or opposite spin, while SSC is much more effective for opposite-spin band pairs.
\end{abstract}

DOI: 10.1103/PhysRevB.103.L041111

Topologically protected electronic modes are seen as an important ingredient in next-generation electronic devices for low-loss electronics and in new approaches towards quantum computation [1,2]. The existence of these is intertwined with the emergent Berry curvature field $\boldsymbol{\Omega}_{\mathbf{k}}$ acting on the quantummechanical wave functions. For example, recent experiments on topological (semi)metallic states in collinear ferromagnets [3-7] have established a connection between Fermi arc or drumhead surface states and excess Berry curvature in the bulk, detectable through the anomalous Hall conductivity $\sigma_{x y}^{\mathrm{A}}=-\left(e^{2} / h\right) \int d^{3} k \Omega_{\mathbf{k}}^{z} /(2 \pi)^{3}$. It is noted that theoretical work has consistently emphasized the role of spin-orbit coupling (SOC) in magnetic topological (semi)metals, which is believed to provide the most direct route to generating nontrivial bulk states. At the same time, calculations show that noncoplanar magnetism with nonzero scalar spin chirality (SSC) $\chi_{i j k}=\mathbf{m}_{i} \cdot\left(\mathbf{m}_{j} \times \mathbf{m}_{k}\right)$ of three neighboring moments $i, j, k$ can play a conceptually very similar role [8-10], leading to band mixing, Berry curvature, and a sideways deflection of moving charge carriers termed the topological (or geometrical) Hall effect. This Hall signal is often associated with skyrmion lattice states [11-14].

In the present work, we highlight a fundamental difference between Berry curvature $\boldsymbol{\Omega}_{\mathbf{k}}$ generated by SOC and SSC [8-10]. For this purpose, we separate contributions to the $\boldsymbol{\Omega}_{\mathbf{k}}$ from band pairs $n \neq m$ with opposite and equal spins as $\boldsymbol{\Omega}_{\mathbf{k}}^{\text {diff. }}=\sum_{n, m} w_{n m}^{\text {diff. }} \boldsymbol{\Omega}_{\mathbf{k}}^{n m}$ and $\boldsymbol{\Omega}_{\mathbf{k}}^{\text {same }}=$

\footnotetext{
*hirschberger@ap.t.u-tokyo.ac.jp

†Present address: Paul-Scherrer-Institute, 5232 Villigen PSI, Switzerland.
}

$\sum_{n, m} w_{n m}^{\text {same }} \boldsymbol{\Omega}_{\mathbf{k}}^{n m}$, respectively. Our main result is that SOC in collinear ferromagnets [3,15-19] mixes bands of equal and opposite spins, giving comparable amplitudes of both $\boldsymbol{\Omega}_{\mathbf{k}}^{\text {same }}$ and $\boldsymbol{\Omega}_{\mathbf{k}}^{\text {diff. }}$ [20]. In contrast, the synthetic gauge field from noncoplanar magnetic order with $\chi_{i j k} \neq 0$ is related mostly to $\boldsymbol{\Omega}_{\mathbf{k}}^{\text {diff. }}$. In this scenario, large $\boldsymbol{\Omega}_{\mathbf{k}}^{\text {diff. }}$ originates at (near-)degeneracy points for bands of opposite spin.

The Hall signals from SOC and SSC are often referred to as Karplus-Luttinger-type [21] anomalous Hall conductivity $\sigma_{x y}^{\mathrm{KL}}$ and as the geometrical or topological Hall effect $\sigma_{x y}^{\mathrm{G}}$, respectively. Given a spin texture $\mathbf{m}_{i}$ of the conducting states, where $i$ labels discrete lattice sites, the leading terms on symmetry grounds [22] are $\sigma_{x y}^{\mathrm{KL}} \sim \sum_{i} m_{i}^{z}$ and $\sigma_{x y}^{\mathrm{G}} \sim \sum_{\langle i j k\rangle} \chi_{i j k}$. The sum $\langle i j k\rangle$ is over nearest-neighbor spin sites [see Fig. 1(b)]. Our combined experimental and theoretical investigation provides a unified picture of Berry curvature in momentum space, comprising both these signals.

The target material of this study is the metallic pyrochlore $\mathrm{Nd}_{2} \mathrm{Mo}_{2} \mathrm{O}_{7}$, a canonical canted ferromagnet [23-26]. Its crystal structure is composed of interpenetrating tetrahedra hosting, in turn, $\mathrm{Mo}^{4+}$ and $\mathrm{Nd}^{3+}$ magnetic moments. The spin arrangement on Mo tetrahedra for two magnetic phases is illustrated in Figs. 1(a)-1(c), demonstrating that the band picture is a reasonable starting point for this material where SSC appears with a short characteristic length scale $\lambda \leqslant a$. Here, $a$ is the lattice constant of the cubic pyrochlore structure. A large body of literature has focused on continuous spin textures with $\lambda>a$, such as skyrmions in noncentrosymmetric magnets. For such very large spin textures it is more appropriate to consider semiclassical transport under the influence of a Berry curvature field living in real space $[11,13,14,27]$. 

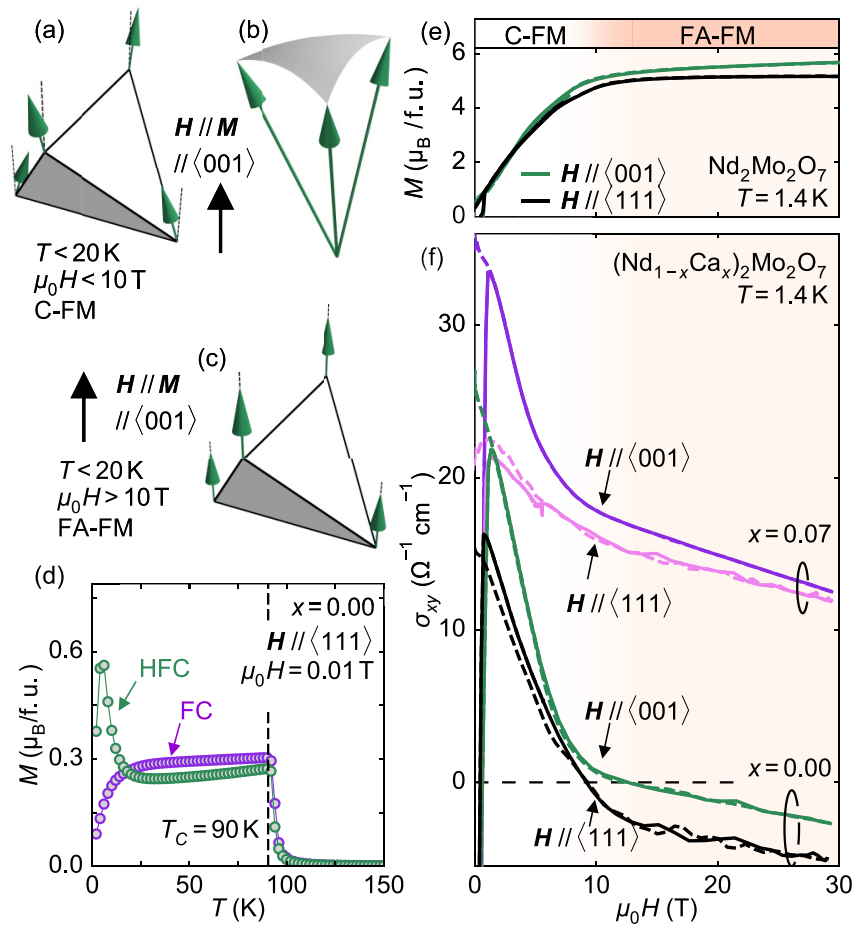

FIG. 1. Smooth transition of the molybdenum sublattice in $\left(\mathrm{Nd}_{1-x} \mathrm{Ca}_{x}\right)_{2} \mathrm{Mo}_{2} \mathrm{O}_{7}$ (green arrows) from (a) and (b) canted ferromagnet (C-FM) at low field to (c) field-aligned ferromagnet (FA-FM). (d) Magnetization $M$ as a function of increasing temperature $T$ at $x=0.00$ after preparation in a field-cooled (FC) or high-field-cooled (HFC) state. Ferromagnetic ordering on the molybdenum sublattice at $T_{C}$ is marked by a dashed line. (e) and (f) High-field magnetization and Hall conductivity measurements. The anisotropy of transport and magnetic properties is exemplified using two directions of the magnetic field H. In (e) and (f), curves for increasing and decreasing magnetic field are marked by solid and dashed lines, while white and orange shaded regions in (e) and (f) indicate the C-FM and FA-FM regimes, respectively.

The ferromagnetic transition of the Mo sublattice at $T_{C}=$ $90 \mathrm{~K}$ is driven by the double-exchange mechanism (step anomaly in Fig. 1(d); see Ref. [28]). Meanwhile, the $\mathrm{Nd}^{3+}$ sublattice takes a back seat at first and, upon further cooling, undergoes a smooth crossover towards long-range order with a two-in, two-out configuration on individual rare-earth tetrahedra $[23,25,26]$. This crossover at $T^{*} \sim 20 \mathrm{~K}$ is related to rapid growth of the coercive field, visible as a departure of magnetization curves $M(T)$ recorded after cooling in $\mu_{0} H=1 \mathrm{~T}$ (high field cooled) and in $\mu_{0} H=0.01 \mathrm{~T}$ [field cooled; see Fig. 1(d)]. The noncoplanar alignment of the $\mathrm{Nd}^{3+}$ moments is transmitted to the conducting Mo states by moderate $d-f$ coupling, so that the Mo sublattice ultimately adopts canted ferromagnetic (C-FM) order with a tilting angle of $\sim 5^{\circ}-15^{\circ}$ away from the preferred $\langle 001\rangle$ easy direction (Figs. 1(a) and 1(b) and Refs. [23,25]).

Both noncoplanar and collinear arrangements of molybdenum spins are experimentally accessible in $\mathrm{Nd}_{2} \mathrm{Mo}_{2} \mathrm{O}_{7}$. By using a high magnetic field, we are able to fully align the Mo $4 d$ moments and reach a field-aligned ferromagnetic (FA-FM) state at low temperature [orange shading in Figs. 1(e) and 1(f)]. To describe the magnetization isotherms in Fig. 1(e), the net magnetization is decomposed into a sum of two contributions from Mo and Nd sublattices, $\mathbf{M}=\mathbf{M}_{\mathrm{Nd}}+\mathbf{M}_{\mathrm{Mo}}$. The small size of the spontaneous moment, $M_{0}=0.3 \mu_{\mathrm{B}} / \mathrm{f}$.u., is caused by near-complete cancellation between antiferromagnetically coupled $\mathbf{M}_{\mathrm{Nd}}$ and $\mathbf{M}_{\mathrm{Mo}}$ in zero field [24]. However, the neodymium moments can be coaligned with $\mathbf{M}_{\mathbf{M o}}$ in $\mu_{0} H \sim 10 \mathrm{~T}$, and it is understood that the canting of Mo spins is completely suppressed at $\mu_{0} H>10 \mathrm{~T}$ [24-26]. Note that even in FA-FM, $\mathrm{Nd}^{3+}$ are not fully parallel to $\mathbf{H}$ up to fields $>100 \mathrm{~T}$.

Figure 1(f) (black and green curves) demonstrates that CFM and FA-FM regimes of $\mathrm{Nd}_{2} \mathrm{Mo}_{2} \mathrm{O}_{7}$ have distinct transport signatures, with much larger Hall conductivity $\sigma_{x y}$ in C-FM [Figs. 1(a) and 1(b)]. The Hall signal is sharply suppressed at $\mu_{0} H>10 \mathrm{~T}$ when the Mo spins are coaligned [24]. For further analysis of the transport properties, let us separate $\sigma_{x y}=$ $\sigma_{x y}^{\mathrm{N}}+\sigma_{x y}^{\mathrm{A}}$, where $\sigma_{x y}^{\mathrm{N}}$ and $\sigma_{x y}^{\mathrm{A}}=\sigma_{x y}^{\mathrm{KL}}+\sigma_{x y}^{\mathrm{G}}$ are the normal and anomalous Hall conductivities, respectively. In $\mathrm{Nd}_{2} \mathrm{Mo}_{2} \mathrm{O}_{7}$, we have $\sigma_{x y}^{\mathrm{N}} \propto H$ from the orbital motion of electrons and $\sigma_{x y}^{\mathrm{KL}} \propto M_{\mathrm{Mo}}$ for the SOC-driven signal. Meanwhile, $\sigma_{x y}^{\mathrm{G}}$ has strong field dependence as the spin umbrella of the C-FM order is continuously closed with increasing field [Figs. 1(a) and 1(c)]. We focus the main part of our discussion on the experimental geometry where $\mathbf{H} / /\langle 001\rangle$ but also note that the Hall conductivity shows considerable anisotropy at low magnetic fields [Fig. 1(f)]. As discussed in the Supplemental Material (SM) [29], the low-field $\sigma_{x y}$ in the configuration $\mathbf{H} / /\langle 111\rangle$ is suppressed due to the effect of projecting the vector $\boldsymbol{\Omega}_{\mathbf{k}}$ onto the $x-y$ plane defined by the voltage and current contacts.

The heart of our study is the experimental control of the Fermi energy $\varepsilon_{F}$ in $\mathrm{Nd}_{2} \mathrm{Mo}_{2} \mathrm{O}_{7}$ by chemical substitution of $\mathrm{Ca}^{2+}$ for $\mathrm{Nd}^{3+}$ in $\left(\mathrm{Nd}_{1-x} \mathrm{Ca}_{x}\right)_{2} \mathrm{Mo}_{2} \mathrm{O}_{7}$ (NCMO) and a comparison of the observed transport response with first-principles calculations. In the SM it is shown that the magnetic ground state C-FM of this compound is robust up to $x \approx 0.15$ ( $x=$ 0.30 for the FA-FM state), justifying our subsequent focus on the band-filling effect. Figure 1(f) includes Hall effect measurements for crystals where $\varepsilon_{F}$ is shifted downwards (hole doping, $x=0.07$, violet and magenta curves). While this batch has nearly identical $T_{C}$ and longitudinal resistivity compared to $x=0$ [29], $\sigma_{x y}(H)$ is now strongly modified; slightly weakened low-field $\sigma_{x y}^{\mathrm{G}}$ contrasts with significantly enhanced $\sigma_{x y}^{\mathrm{KL}}$. The high-field slope of $\sigma_{x y}$, i.e., $\sigma_{x y}^{\mathrm{N}}$, remains nearly unchanged.

The anomalous Hall conductivity $\sigma_{x y}^{\mathrm{A}}=\sigma_{x y}^{\mathrm{KL}}+\sigma_{x y}^{\mathrm{G}}$ (after separation of $\sigma_{x y}^{\mathrm{N}}$ ) is shown in Fig. 2. As the canting angle of Mo spins is only $\sim 10^{\circ}$, the dependence of $M_{\text {Mo }}$ on magnetic field is rather weak, and extrapolation from the FA-FM regime to zero field is suitable for separation of the geometrical Hall signal. We mark $\sigma_{x y}^{\mathrm{KL}} \sim M_{\mathrm{Mo}}$ by violet shading in Fig. 2. Values for the SOC- and SSC-driven Hall conductivities are thus obtained as a function of band filling $x$ [Figs. 3(a)-3(c)] Note that $\sigma_{x y}^{\mathrm{G}}$ vanishes above $x=0.2$ in proximity to a phase transition (see the SM for discussion). In contrast the FA-FM state is realized in high fields even at elevated $x$, so that Fig. 3(b) includes data for $\sigma_{x y}^{\mathrm{KL}}$ up to $x=0.22$. Figure 3(a) also reports the measured low-field total Hall conductivity 

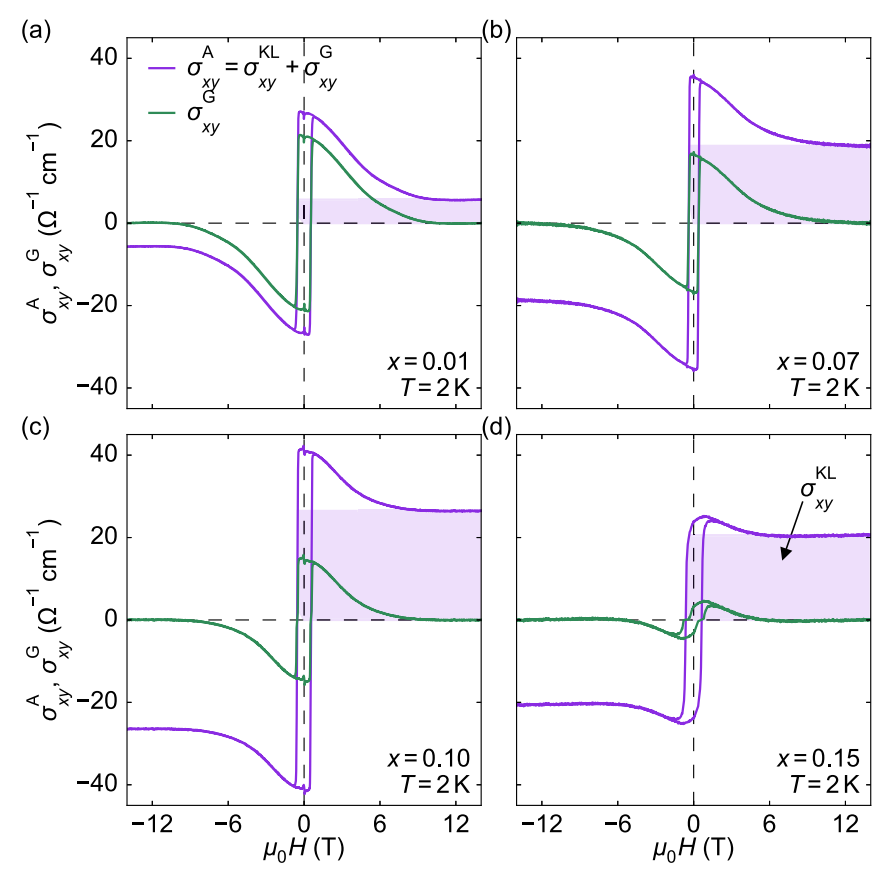

FIG. 2. Separation of Hall conductivities in $\mathrm{Nd}_{2} \mathrm{Mo}_{2} \mathrm{O}_{7}$ at $T=$ $2 \mathrm{~K}$ and $\mathbf{H} / /\langle 001\rangle$. We show the anomalous Hall conductivity $\sigma_{x y}^{\mathrm{A}}$ (violet curves) after subtraction of the field-linear normal Hall signal and the geometrical Hall signal $\sigma_{x y}^{\mathrm{G}}$ generated by SSC (green curves). The Hall signal originating from spin-orbit coupling $\sigma_{x y}^{\mathrm{KL}}$ is marked by a violet shaded box in each plot.

$\sigma_{x y}^{0.5 \mathrm{~T}} \approx \sigma_{x y}^{\mathrm{A}}=\sigma_{x y}^{\mathrm{KL}}+\sigma_{x y}^{\mathrm{G}}$ for two directions of the magnetic field (violet and green symbols). As noted above and in the SM, the low-field anisotropy arises due to a slight preference of Mo spins for the crystallographic $\langle 001\rangle$ axis.

The overall band-filling dependence of $\sigma_{x y}^{\mathrm{KL}}$ and $\sigma_{x y}^{\mathrm{G}}$ can be reproduced in the framework of density functional theory. We start from the band structure in the collinear ferromagnetic state of $\mathrm{NCMO}(x=0)$, where the size of the ordered moment is set to about $1.5 \mu_{B}$ /Mo [29], consistent with the experimental estimate [23-26]. Electronic bands of molybdenum $4 d$ character dominate at the Fermi edge in this calculation, and both SOC and SSC modulate the electronic structure around $\varepsilon_{F}$, giving rise to finite $\boldsymbol{\Omega}_{\mathbf{k}}$ and $\sigma_{x y}^{\mathrm{A}}$. Further changing $\varepsilon_{F}$ in the rigid-band approximation yields the filling dependence of

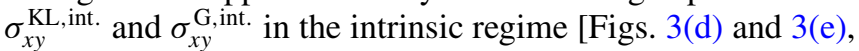
left-hand $y$ axes]. For comparison to the real material, the effect of quenched disorder must be taken into account using

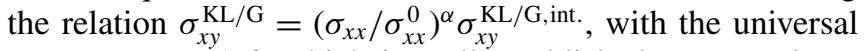
exponent $\alpha=1.6$, which is well established across a large number of material classes $[22,29,30]$. Here $\sigma_{x x}^{0}$ is the threshold conductivity value above which the compound enters the intrinsic regime with dissipationless anomalous Hall current. While empirically $\sigma_{x x}^{0} \approx 10^{4} \Omega^{-1} \mathrm{~cm}^{-1}$ [30], we tentatively use the value $0.6 \times 10^{4} \Omega^{-1} \mathrm{~cm}^{-1}$ to get a good fit between theory and experiment for the Karplus-Luttinger term. The same correction procedure (same $\alpha$ ) is applied to $\sigma_{x y}^{\mathrm{G}, \text { int., as }}$ shown in Fig. 3(e). Further details and the possibility of slightly different $\alpha$ for $\sigma_{x y}^{\mathrm{G}}$ and $\sigma_{x y}^{\mathrm{KL}}$ are considered in the SM.

Calculation and experiment show a dramatic rise in $\sigma_{x y}^{\mathrm{KL}}$ when increasing the hole content above $x=0$, followed by a
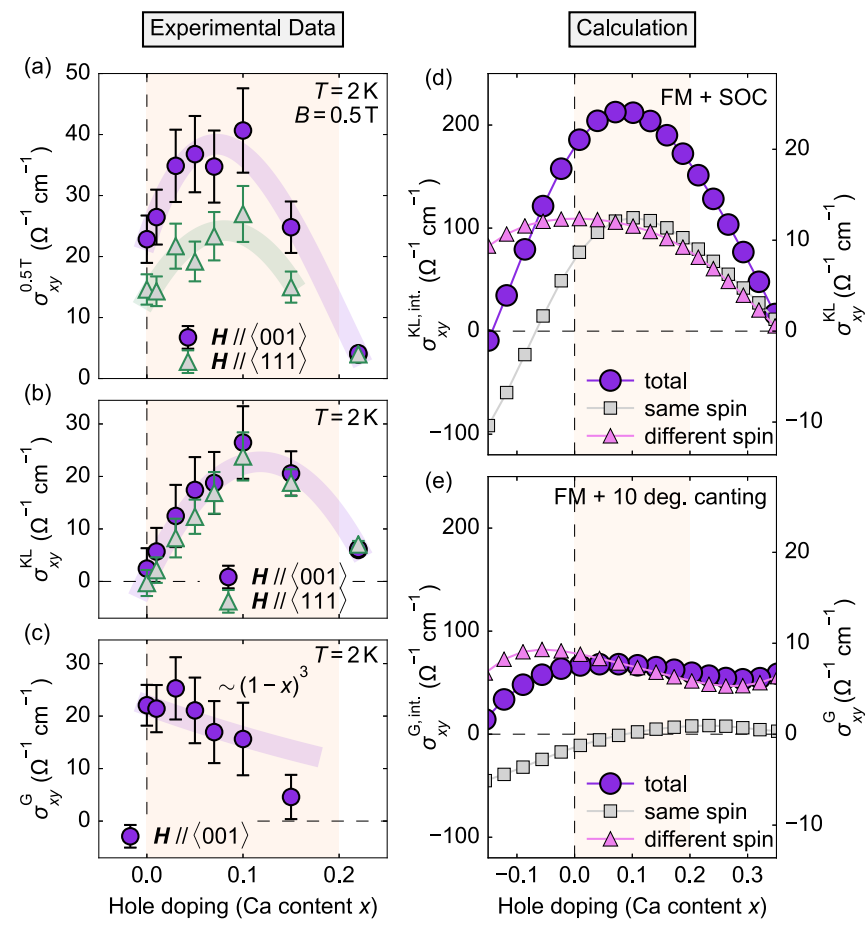

FIG. 3. (a) Low-field Hall conductivity $\sigma_{x y}^{0.5 \mathrm{~T}}$ in $\left(\mathrm{Nd}_{1-x} \mathrm{Ca}_{x}\right)_{2} \mathrm{Mo}_{2} \mathrm{O}_{7}$, a proxy for the anomalous Hall signal $\sigma_{x y}^{\mathrm{A}}=\sigma_{x y}^{\mathrm{G}}+\sigma_{x y}^{\mathrm{KL}}$. (b)-(e) Measured and calculated SOC-driven Hall conductivity $\sigma_{x y}^{\mathrm{KL}}$ as well as SSC-driven geometrical Hall conductivity $\sigma_{x y}^{\mathrm{G}}$. In (d) and (e), a second $y$ axis indicates the result of the calculation $\left(\sigma_{x y}^{\mathrm{KL}, \text { int. }}\right.$ and $\sigma_{x y}^{\mathrm{G}, \text { int }}$, see text) before applying the universal correction for finite defect concentration. Moreover, contributions to $\sigma_{x y}^{\mathrm{KL}}$ and $\sigma_{x y}^{\mathrm{G}}$ from equal-spin and opposite-spin Berry curvatures $\left(\boldsymbol{\Omega}_{\mathbf{k}}^{\text {same }}\right.$ and $\left.\boldsymbol{\Omega}_{\mathbf{k}}^{\text {diff. }}\right)$ are displayed separately. Thick magenta and green lines in (a) and (b) are guides to the eye. Error bars in (a)-(c) are calculated from the estimated sample geometry error $(\sim \pm 17 \%)$. In (c), the thick purple line indicates the $(1-x)^{3}$ power law expected from dilution of rare-earth magnetic moments (see text).

drop when $x \geqslant 0.10$ [Figs. 3(b) and 3(d)]. The experimentally observed $\sigma_{x y}^{\mathrm{G}}$ is gently suppressed with $x$, roughly following $\sigma_{x y}^{\mathrm{G}} \sim(1-x)^{3}$. This phenomenological law [31] describes the reduction of SSC due to the dilution of rare-earth moments with nonmagnetic $\mathrm{Ca}^{2+}$. Discounting the dilution effect, we conclude that the numerical and experimental data for $\sigma_{x y}^{\mathrm{G}}$ both indicate very mild $x$ dependence for $x<0.15$. We stress that these calculations reproduce the sign of two components of the anomalous Hall signal, their order of magnitude, the resonance-type increase and decrease of $\sigma_{x y}^{\mathrm{KL}}$ as a function of $x$, and the rather mild band-filling dependence of $\sigma_{x y}^{\mathrm{G}}$. While the former experiences a maximum around $x=0.1$ in both experiment and theory, the experimental $\sigma_{x y}^{\mathrm{KL}}$ peak is narrower than that of the calculations, as seen in Figs. 3(b) and 3(d). This squeezing phenomenon can be attributed, perhaps, to the electron correlation effect, which is not captured in the present theory [29]. A decomposition of calculated Hall conductivities in terms of band pairs of equal spin and band pairs of opposite spin, i.e., in terms of $\boldsymbol{\Omega}_{\mathbf{k}}^{\text {same }}$ and $\boldsymbol{\Omega}_{\mathbf{k}}^{\text {diff. }}$, is shown in Figs. 3(d) and 3(e). While $\sigma_{x y}^{\mathrm{KL}}$ has roughly equal 

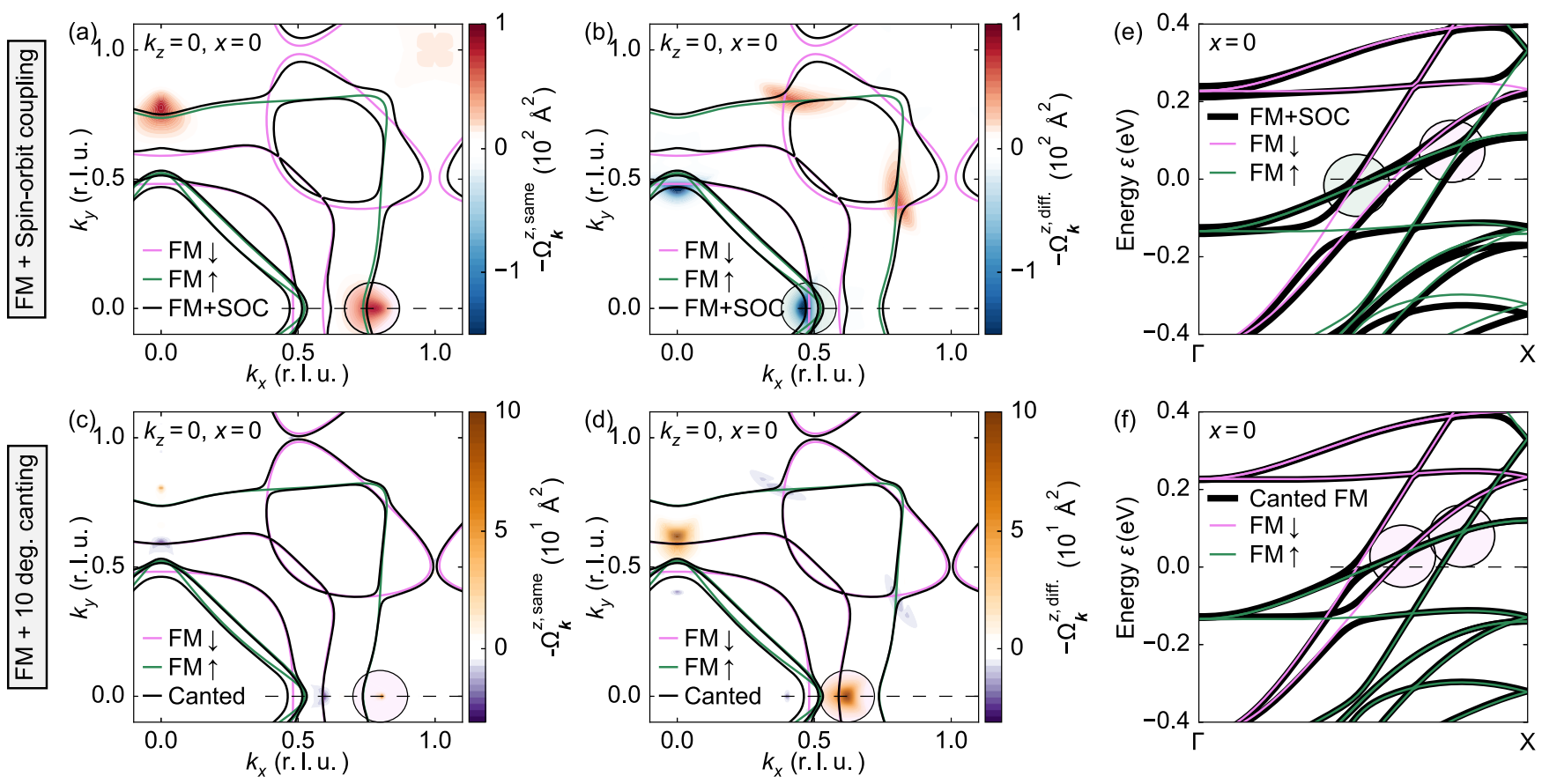

FIG. 4. (a)-(d) Contour maps of Berry curvature $-\Omega_{\mathbf{k}}^{z}$ in the $k_{z}=0$ plane, where same-spin and opposite-spin contributions, as defined in the text, are shown separately in two columns. The top row is for the collinear ferromagnetic state with spin-orbit coupling (SOC); the bottom row is for the canted ferromagnetic (C-FM) state without SOC. Black curves indicate Fermi surface cross sections. For comparison, cross-sectional lines of the minority (FM- $\downarrow$ ) and majority (FM- $\uparrow$ ) Fermi surfaces in the collinear state, without SOC, are also shown. The direction of the $\Gamma-X$ line in reciprocal space is indicated by a dashed line. (e) and (f) Along $\Gamma$ - $X$, anticrossing points of electronic bands are present both in the ferromagnetic state with SOC and in the C-FM state without SOC. In magenta and green, ferromagnetic bands (no SOC) are plotted for comparison. Some band-anticrossing points can be directly associated with features in the $-\Omega_{\mathbf{k}}^{z}$ maps (highlighted by shaded circles). Dashed lines mark the position of the Fermi energy $\varepsilon_{F}$.

contributions from these two, $\sigma_{x y}^{\mathrm{G}}$ is dominated by the latter. Hence, the canted spin structure allows strong hybridization of bands with mostly up and mostly down spins but does not affect band pairs with equal spin to leading order.

We illustrate the momentum-space distribution of $\Omega_{\mathbf{k}}^{z}$ along two-dimensional cuts of the fcc cubic Brillouin zone (BZ) with $k_{z}=0$ in Figs. 4(a)-4(d). The contributions $\Omega_{\mathbf{k}}^{\text {same, } z}$ and $\Omega_{\mathbf{k}}^{\text {diff.,z }}$ are plotted separately, and the $z$ axis is parallel to $\langle 001\rangle$. These contour plots, together with line cuts along the $\Gamma-X$ direction in momentum space [Figs. 4(e) and 4(f)], provide an example of how SSC and SOC generate $\Omega_{\mathbf{k}}^{z}$ at different points in the BZ and sometimes even cause opposite signs of $\Omega_{\mathbf{k}}^{z}$ in the same region of the BZ. The high sensitivity of the geometrical Hall signal $\sigma_{x y}^{\mathrm{G}}$ to the spin character of electronic bands is in sharp contrast to the case of the SOC-induced $\sigma_{x y}^{\mathrm{KL}}$. It follows that (nearly) half-metallic magnets with SSC, i.e., with conduction bands exclusively of the spin-down (magnetic moment up, FM- $\uparrow$ ) type, are not well suited for the observation of enhanced $\sigma_{x y}^{\mathrm{G}}$. Thus, the present work gives guidance in the search for large and possibly quantized geometrical Hall responses due to SSC: The combination of complex magnetism with opposite-spin band crossings near the Fermi energy is expected to be a powerful predictor in the search for giant geometrical (or topological) Hall conductivity.

Acknowledgments. We have benefited from discussions with N. Nagaosa, K. Ueda, I. Belopolski, and J. Masell. M.H. was supported as a Humboldt/JSPS International Research Fellow (18F18804). Y.N. acknowledges JSPS KAKENHI Grants-in-Aid for Scientific Research (Grants No. 16H06345, No. 17K14336, and No. 18H01158). L.S. was funded by the German Academic Exchange Service (DAAD) via a PROMOS scholarship awarded by the German Federal Ministry of Education and Research (BMBF). This work was partially supported by JST CREST Grant No. JPMJCR1874 (Japan).

M.H. and Y.N. contributed equally to this work.
[1] M. Z. Hasan and C. L. Kane, Colloquium: Topological insulators, Rev. Mod. Phys. 82, 3045 (2010).

[2] B. Yan and C. Felser, Topological materials: Weyl semimetals, Annu. Rev. Condens. Matter Phys. 8, 337 (2017).
[3] L. Ye, M. Kang, J. Liu, F. von Cube, C. R. Wicker, T. Suzuki, C. Jozwiak, A. Bostwick, E. Rotenberg, D. C. Bell, L. Fu, R. Comin, and J. G. Checkelsky, Massive Dirac fermions in a ferromagnetic kagome metal, Nature (London) 555, 638 (2018). 
[4] N. Morali, R. Batabyal, P. Kumar Nag, E. Liu, Q. Xu, Y. Sun, B. Yan, C. Felser, N. Avraham, and H. Beidenkopf, Fermi-arc diversity on surface terminations of the magnetic Weyl semimetal $\mathrm{Co}_{3} \mathrm{Sn}_{2} \mathrm{~S}_{2}$, Science 365, 1286 (2019).

[5] I. Belopolski et al., Discovery of topological Weyl fermion lines and drumhead surface states in a room temperature magnet, Science 365, 1278 (2019).

[6] D. F. Liu, A. J. Liang, E. K. Liu, Q. N. Xu, Y. W. Li, C. Chen, D. Pei, W. J. Shi, S. K. Mo, P. Dudin, T. Kim, C. Cacho, G. Li, Y. Sun, L. X. Yang, Z. K. Liu, S. S. P. Parkin, C. Felser, and Y. L. Chen, Magnetic Weyl semimetal phase in a Kagomé crystal, Science 365, 1282 (2019).

[7] J.-X. Yin et al., Quantum-limit Chern topological magnetism in $\mathrm{TbMn}_{6} \mathrm{Sn}_{6}$, Nature (London) 583, 533 (2020).

[8] K. Ohgushi, S. Murakami, and N. Nagaosa, Spin anisotropy and quantum Hall effect in the kagomé lattice: Chiral spin state based on a ferromagnet, Phys. Rev. B 62, R6065(R) (2000).

[9] R. Shindou and N. Nagaosa, Orbital Ferromagnetism and Anomalous Hall Effect in Antiferromagnets on the Distorted fcc Lattice, Phys. Rev. Lett. 87, 116801 (2001).

[10] I. Martin and C. D. Batista, Itinerant Electron-Driven Chiral Magnetic Ordering and Spontaneous Quantum Hall Effect in Triangular Lattice Models, Phys. Rev. Lett. 101, 156402 (2008).

[11] P. Bruno, V. K. Dugaev, and M. Taillefumier, Topological Hall Effect and Berry Phase in Magnetic Nanostructures, Phys. Rev. Lett. 93, 096806 (2004).

[12] M. Lee, W. Kang, Y. Onose, Y. Tokura, and N. P. Ong, Unusual Hall Effect Anomaly in MnSi under Pressure, Phys. Rev. Lett. 102, 186601 (2009).

[13] A. Neubauer, C. Pfleiderer, B. Binz, A. Rosch, R. Ritz, P. G. Niklowitz, and P. Böni, Topological Hall Effect in the $A$ Phase of MnSi, Phys. Rev. Lett. 102, 186602 (2009).

[14] R. Ritz, M. Halder, C. Franz, A. Bauer, M. Wagner, R. Bamler, A. Rosch, and C. Pfleiderer, Giant generic topological Hall resistivity of MnSi under pressure, Phys. Rev. B 87, 134424 (2013).

[15] Z. Fang, N. Nagaosa, K. S. Takahashi, A. Asamitsu, R. Mathieu, T. Ogasawara, H. Yamada, M. Kawasaki, Y. Tokura, and K. Terakura, The anomalous Hall effect and magnetic monopoles in momentum space, Science 302, 92 (2003).

[16] E. Liu et al., Giant anomalous Hall effect in a ferromagnetic Kagome-lattice semimetal, Nat. Phys. 14, 1125 (2018).

[17] G. Chang et al., Magnetic and noncentrosymmetric Weyl fermion semimetals in the $R$ AlGe family of compounds $(R=$ rare earth), Phys. Rev. B 97, 041104(R) (2018).

[18] D. Gosálbez-Martínez, I. Souza, and D. Vanderbilt, Chiral degeneracies and Fermi-surface Chern numbers in bcc Fe, Phys. Rev. B 92, 085138 (2015).

[19] Q. Xu, E. Liu, W. Shi, L. Muechler, J. Gayles, C. Felser, and Y. Sun, Topological surface Fermi arcs in the magnetic Weyl semimetal $\mathrm{Co}_{3} \mathrm{Sn}_{2} \mathrm{~S}_{2}$, Phys. Rev. B 97, 235416 (2018).

[20] X. Wang, J. R. Yates, I. Souza, and D. Vanderbilt, Ab initio calculation of the anomalous Hall conductivity by Wannier interpolation, Phys. Rev. B 74, 195118 (2006).

[21] R. Karplus and J. M. Luttinger, Hall effect in ferromagnetics, Phys. Rev. 95, 1154 (1954).

[22] N. Nagaosa, J. Sinova, S. Onoda, A. H. MacDonald, and N. P. Ong, Anomalous Hall effect, Rev. Mod. Phys. 82, 1539 (2010).
[23] Y. Taguchi, Y. Oohara, H. Yoshizawa, N. Nagaosa, and Y. Tokura, Spin chirality, Berry phase, and anomalous Hall effect in a frustrated ferromagnet, Science 291, 2573 (2001).

[24] Y. Taguchi, T. Sasaki, S. Awaji, Y. Iwasa, T. Tayama, T. Sakakibara, S. Iguchi, T. Ito, and Y. Tokura, Magnetic Field Induced Sign Reversal of the Anomalous Hall Effect in a Pyrochlore Ferromagnet $\mathrm{Nd}_{2} \mathrm{Mo}_{2} \mathrm{O}_{7}$ : Evidence for a Spin Chirality Mechanism, Phys. Rev. Lett. 90, 257202 (2003).

[25] Y. Yasui, S. Iikubo, H. Harashina, T. Kageyama, M. Ito, M. Sato, and K. Kakurai, Neutron scattering studies of pyrochlore compound $\mathrm{Nd}_{2} \mathrm{Mo}_{2} \mathrm{O}_{7}$ in magnetic field, J. Phys. Soc. Jpn 72, 865 (2003).

[26] Y. Yasui, T. Kageyama, T. Moyoshi, M. Soda, M. Sato, and K. Kakurai, Studies of anomalous Hall effect and magnetic structure of $\mathrm{Nd}_{2} \mathrm{Mo}_{2} \mathrm{O}_{7}$ : Test of chirality mechanism, J. Phys. Soc. Jpn. 75, 084711 (2006).

[27] J. Ye, Y. B. Kim, A. J. Millis, B. I. Shraiman, P. Majumdar, and Z. Tešanović, Berry Phase Theory of the Anomalous Hall Effect: Application to Colossal Magnetoresistance Manganites, Phys. Rev. Lett. 83, 3737 (1999).

[28] T. Katsufuji, H. Y. Hwang, and S.-W. Cheong, Anomalous Magnetotransport Properties of $\mathrm{Nd}_{2} \mathrm{Mo}_{2} \mathrm{O}_{7}$ near the Magnetic Phase Boundary, Phys. Rev. Lett. 84, 1998 (2000).

[29] See Supplemental Material at http://link.aps.org/supplemental/ 10.1103/PhysRevB.103.L041111 for details of theoretical calculations and experimental transport and magnetization measurements (as well as a discussion of the magnetic phase diagram), which includes Refs. [32-49].

[30] S. Onoda, N. Sugimoto, and N. Nagaosa, Quantum transport theory of anomalous electric, thermoelectric, and thermal Hall effects in ferromagnets, Phys. Rev. B 77, 165103 (2008).

[31] K. Ueda, S. Iguchi, T. Suzuki, S. Ishiwata, Y. Taguchi, and Y. Tokura, Topological Hall Effect in Pyrochlore Lattice with Varying Density of Spin Chirality, Phys. Rev. Lett. 108, 156601 (2012).

[32] P. Giannozzi et al., Advanced capabilities for materials modeling with QUANTUM ESPRESSO, J. Phys.: Condens. Matter 29, 465901 (2017).

[33] Y. Moritomo, Sh. Xu, A. Machida, T. Katsufuji, E. Nishibori, M. Takata, M. Sakata, and S.-W. Cheong, Chemical pressure control of exchange interaction in Mo pyrochlore, Phys. Rev. B 63, 144425 (2001).

[34] J. P. Perdew, K. Burke, and M. Ernzerhof, Generalized Gradient Approximation Made Simple, Phys. Rev. Lett. 77, 3865 (1996).

[35] A. Dal Corso, Pseudopotentials periodic table: From $\mathrm{H}$ to $\mathrm{Pu}$, Comput. Mater. Sci. 95, 337 (2014).

[36] N. Marzari and D. Vanderbilt, Maximally localized generalized Wannier functions for composite energy bands, Phys. Rev. B 56, 12847 (1997).

[37] I. Souza, N. Marzari, and D. Vanderbilt, Maximally localized Wannier functions for entangled energy bands, Phys. Rev. B 65, 035109 (2001).

[38] P. Pizzi et al., Wannier90 as a community code: New features and applications, J. Phys.: Condens. Matter 32, 165902 (2020).

[39] M. G. Lopez, D. Vanderbilt, T. Thonhauser, and I. Souza, Wannier-based calculation of the orbital magnetization in crystals, Phys. Rev. B 85, 014435 (2012). 
[40] F. R. Lux, F. Freimuth, S. Blügel, and Y. Mokrousov, Chiral Hall Effect in Noncollinear Magnets from a Cyclic Cohomology Approach, Phys. Rev. Lett. 124, 096602 (2020).

[41] J. Kipp, K. Samanta, F. R. Lux, M. Merte, J.-P. Hanke, M. Redies, F. Freimuth, S. Blügel, M. Ležaić, and Y. Mokrousov, The chiral Hall effect in canted ferromagnets and antiferromagnets, arXiv:2007.01529.

[42] T. Miyasato, N. Abe, T. Fujii, A. Asamitsu, S. Onoda, Y. Onose, N. Nagaosa, and Y. Tokura, Crossover Behavior of the Anomalous Hall Effect and Anomalous Nernst Effect in Itinerant Ferromagnets, Phys. Rev. Lett. 99, 086602 (2007).

[43] T. Suzuki, R. Chisnell, A. Devarakonda, Y.-T. Liu, W. Feng, D. Xiao, J. W. Lynn, and J. G. Checkelsky, Large anomalous Hall effect in a half-Heusler antiferromagnet, Nat. Phys. 12, 1119 (2016).

[44] G. Kotliar and D. Vollhardt, Strongly correlated materials: Insights from dynamical mean-field theory, Phys. Today 57(3), 53 (2004).
[45] Y. Kaneko and Y. Tokura, Floating zone furnace equipped with a high power laser of $1 \mathrm{~kW}$ composed of five smart beams, J. Cryst. Growth 533, 125435 (2020).

[46] A. Apetrei, I. Mirebeau, I. Goncharenko, D. Andreica, and P. Bonville, Microscopic Study of a Pressure-Induced Ferromagnetic-Spin-Glass Transition in the Geometrically Frustrated Pyrochlore $\left(\mathrm{Tb}_{1-x} \mathrm{La}_{x}\right)_{2} \mathrm{Mo}_{2} \mathrm{O}_{7}$, Phys. Rev. Lett. 97, 206401 (2006).

[47] I. Mirebeau, A. Apetrei, I. Goncharenko, D. Andreica, P. Bonville, J. P. Sanchez, A. Amato, E. Suard, W. A. Crichton, A. Forget, and D. Colson, Pressure-induced ferromagnet to spinglass transition in $\mathrm{Gd}_{2} \mathrm{Mo}_{2} \mathrm{O}_{7}$, Phys. Rev. B 74, 174414 (2006).

[48] S. Iguchi, N. Hanasaki, M. Kinuhara, N. Takeshita, C. Terakura, Y. Taguchi, H. Takagi, and Y. Tokura, Emergence of a Diffusive Metal State with no Magnetic Order Near the Mott Transition in Frustrated Pyrochlore-Type Molybdates, Phys. Rev. Lett. 102, 136407 (2009).

[49] A. B. Pippard, Magnetoresistance in Metals (Cambridge University Press, Cambridge, 1989). 